\title{
Network analysis of the pharmacological mechanism of Reduning injection in the treatment of novel coronavirus pneumonia
}

\section{Yizi Xie}

The First Clinical College, Guangzhou University of Chinese Medicine

\section{Wenjiang ZHENG}

The First Clinical College, Guangzhou University of Chinese Medicine

\section{Yong JIANG}

Shenzhen Hospital of Integrated Traditional Chinese and Western Medicine

Xiaohong LIU

The First Affiliated Hospital, Guangzhou University of Chinese Medicine

Shaofeng ZHAN ( $\triangle 870557839 @ q q . c o m$ )

\section{Research}

Keywords: coronavirus disease 2019 R eduning injection, network pharmacology, mechanism

Posted Date: June 16th, 2020

DOI: https://doi.org/10.21203/rs.3.rs-34403/v1

License: (9) (i) This work is licensed under a Creative Commons Attribution 4.0 International License.

Read Full License 


\title{
Network analysis of the pharmacological mechanism of Reduning injection in the treatment of novel coronavirus pneumonia
}

\author{
Yizi XIE ${ }^{1}$, Wenjiang ZHENG ${ }^{1}$, Yong JIANG ${ }^{2}$, Xiaohong LIU $^{3}$, Shaofeng ZHAN ${ }^{3}$ \\ ${ }^{1}$ The First Clinical College, Guangzhou University of Chinese Medicine, Guangzhou 510405, China \\ ${ }^{2}$ Shenzhen Hospital of Integrated Traditional Chinese and Western Medicine, Shenzhen 518104, China \\ ${ }^{3}$ The First Affiliated Hospital, Guangzhou University of Chinese Medicine, Guangzhou 510405, China \\ Correspondence should be addressed to Shaofeng ZHAN; zsfstone@126.com
}

\begin{abstract}
Background: Pneumonia caused by novel coronavirus (COVID-19) is spreading worldwide. Traditional Chinese medicines (TCM) have played an important role in COVID-19 treatment in China. This study explores the specific mechanisms of action of Reduning injection (RDN) as a novel clinical treatment for COVID-19.
\end{abstract}

Material and Methods: We collected RDN chemical components and targets, COVID-19-related targets, and predictions of potential targets of RDN treatment for COVID-19 and performed network topology, Gene Ontology (GO) functional enrichment analysis and WikiPathways enrichment analysis of these potential targets.

Results: This study identified 14 chemical components and 200 targets of RDN; 3,558 COVID-19-related targets; 54 potential targets of RDN treatment for COVID-19. GO functional enrichment analysis identified 83 entries in biological process, including regulation of vasoconstriction、 arachidonic acid secretion, and 17 entries in molecular function, including oxidoreductase activity, acting on the aldehyde or oxo group of donors. WikiPathways enrichment analysis identified 16 entries including the interleukin-12 signaling pathway.

Conclusion: In brief, RDN may be involved in the core anti-severe acute respiratory syndrome coronavirus 2 (SARS-CoV-2) action and also in the reduction of the resulting inflammatory response, oxidative stress, and lung tissue damage, and the regulation of apoptosis, providing evidence for the characteristics of TCM in COVID-19 treatment involving multiple components, targets, and pathways.

Key Words: coronavirus disease 2019; Reduning injection; network pharmacology; mechanism 


\section{Introduction}

The outbreak of coronavirus disease 2019 (COVID-19) in December 2019 has gradually spread across China and the world. COVID-19 is caused by the severe acute respiratory syndrome coronavirus 2 (SARS-CoV-2)[1], which is a type of beta-coronavirus of group 2B with a genetic sequence highly homogenous to that of the pathogen of the 2003 SARS outbreak[2]. The common clinical symptoms of COVID-19 include fever, dry cough, and fatigue[3]. Auxiliary examination of patients may also show lymphopenia and bilateral multiple mottling opacities on chest computed tomography (CT)[3]. According to data published by the World Health Organization(WHO) (https://www.who.int/emergencies/diseases/novel-coronavirus-2019), the number of confirmed cases has reached 2,246,291 in 213 countries, areas or territories at the time of this writing (April 20, 2020).

There are no effective antiviral treatments available for COVID-19, which is mainly treated by respiratory support and symptomatic relief regimens[4]. However, traditional Chinese medicines (TCMs) play a very important role in the clinical diagnosis and treatment of COVID-19 in China. TCM formulations for COVID-19 have a unique underlying concept of syndrome differentiation and have achieved good clinical results. TCM may suppress SARS-CoV-2, reduce cytokine storm syndrome, regulate immune response, and promote physical rehabilitation, thus playing a therapeutic role in COVID-19[5].

The proprietary Chinese medicine section of the Diagnosis and Treatment Plan of COVID-19 (trial versions 6 and 7) issued by the National Health Commission of the People's Republic of China proposed that intravenous infusion of $20 \mathrm{ml}$ Reduning injection (RDN) combined with $250 \mathrm{ml} 0.9 \%$ sodium chloride injection twice a day could be applied in severe patients (http://bgs.satcm.gov.cn/zhengcewenjian/2020-03-04/13594.html). The main components of RDN included the herbs of Fructus gardeniae (zhi-zi), Lonicera japonica (Japanese honeysuckle flower, jin-yin-hua), and Artemisia apiacea (qing-hao) for heat-clearing and detoxifying in TCM theory and are used to treat high fever and cough caused by pulmonary infections in clinical practice in China[6]. A previous study has shown that RDN may protect against acute lung injury by inhibiting the AMP-activated protein kinase/mitogen-activated protein kinase/nuclear factor kappa B (AMPK/MAPK/NF- $\mathrm{B}$ ) signaling pathway[7]. RDN also has antiviral effects to reduce the steep Enterovirus 71 (EV71)-induced accumulation of tumor necrosis factor-alpha $(\mathrm{TNF}-\alpha)[6]$. 
The research model of network pharmacology is consistent with the pharmacological characteristics of TCM's multiple components, targets, and action pathways. Application of comprehensive network pharmacology to help determine the molecular mechanisms targeted by TCM promotes TCM from empirical medicine to evidence-based medicine and provides data supporting the extensive use of TCM[8]. In short, this study used a network pharmacology approach to explore the specific mechanism of RDN for COVID-19 treatment.

\section{Materials and Methods}

\subsection{Collection of chemical components and targets of RDN}

The names and chemical abstracts service (CAS) numbers of RDN in the original research literature[9] were collected and cross-checked in the PubChem database to obtain the simplified molecular-input line-entry system (SMILES) files of the compounds. These SMILES files were used to further search for the chemical structure formulas and predict targets by entering the information into the Swiss Target Prediction database (http://www.swisstargetprediction.ch/)[10], as the targets of RDN chemical components.

\subsection{Construction of a chemical component-target network}

The obtained chemical components and targets and their corresponding relationships were imported into the Cytoscape 3.7.0 platform[11] to construct the chemical component-target network map. Through the NetworkAnalyzer plug-in of the Cytoscape 3.7.0 platform[12], the degree values of each node in the network diagrams were calculated. The degree value represents the number of nodes that directly interact with a node in the network; the greater the degree value of a node in a network, the greater the role of this node in the network[13].

\subsection{Collection of targets for COVID-19 and prediction of potential targets of RDN for COVID-19 treatment}

The therapeutic target of COVID-19 was derived from single-cell sequencing data[14]. The angiotensin-converting enzyme 2 (ACE2) gene is the receptor for SARS-CoV-2. ACE2 interacts with the viral spike protein and possibly mediates SARS-CoV-2 infection of alveolar type II cells in the lungs. We downloaded the attachment reported in the literature and identified the genes co-expressed with ACE2 based on the results of single-cell sequencing of colonic epithelial cells. After normalizing the gene names of the original files, the matched human-related targets were obtained as COVID-19 targets. The intersections of the disease targets and the action targets of 
RDN were used as the potential targets for RDN treatment of COVID-19.

\subsection{Network topology analysis of potential targets}

The gene names of the potential targets were entered into the STRING database[15] to obtain the protein-protein interactions (PPIs), which were further imported to the Cytoscape 3.7.0 platform to perform network topology analysis using the Cytohubba plug-in[16] to obtain the PPI network of the targets. The Cytohubba was used to discover the key targets and sub-networks of complex networks. This type of network is meaningful for presenting many types of biological data including PPIs, gene regulations, cellular pathways, and signal transductions. The Cytohubba was used to rank the nodes based on their attributes in the networks to provide 11 topological analysis methods based on the shortest paths, including degree, maximum neighborhood component, edge percolated component [16]. The degree value of a protein is directly related to the importance of it, and proteins with high degree values tend to be key proteins[13]. Hence, the network topology analysis was used to arrange the nodes of the PPI network diagrams in descending order of the degree values to obtain the target ranking in this study.

\subsection{Gene annotation analysis of potential targets}

Gene annotation analysis of potential targets was performed in this study by defaulting to $\mathrm{P}<0.05$ and importing potential targets into the Cytoscope 3.7.0 software, followed by using the GlueGO[17] and GluePedia[18] plug-ins to perform Gene Ontology (GO) functional enrichment analysis[19] and WikiPathways enrichment analysis[20].

\section{Results}

\subsection{Prediction of RDN chemical components and targets}

A total of 14 chemical components of RDN were obtained, such as quercetin and luteolin (see Table 1 for details). 200 targets were obtained from the Swiss Target Prediction database as the targets of RDN chemical components (see Table 2 for details).

TABLE 1: Chemical components of RDN and their CAS number.

\begin{tabular}{ccc}
\hline No. & Chemical component & CAS \\
\hline 1 & syringic acid & $530-57-4$ \\
2 & ferulic acid & $1135-24-6$ \\
3 & benzoic acid & $65-85-0$
\end{tabular}




\begin{tabular}{lcc}
4 & caffeic acid & $331-39-5$ \\
5 & p-hydroxy benzaldehyde & $123-08-0$ \\
6 & vanillin & $121-33-5$ \\
7 & trans-cinnamic acid & $140-10-3$ \\
8 & coumarin & $91-64-5$ \\
9 & isorhamnetin & $480-19-3$ \\
10 & quercetin & $117-39-5$ \\
11 & luteolin & $491-70-3$ \\
12 & rutin & $153-18-4$ \\
13 & hyperoside & $482-36-0$ \\
14 & luteolin-7-O- $\beta-D-$ glucoside & $5373-11-5$ \\
\hline
\end{tabular}

TABLE 2: Targets of RDN.

\begin{tabular}{cccccccccc}
\hline No. & Target & No. & Target & No. & Target & No. & Target & No. & Target \\
\hline 1 & CA2 & 41 & MMP9 & 81 & ADORA2A & 121 & CCNB3 & 161 & CYP1A2 \\
2 & CA7 & 42 & MMP1 & 82 & DAPK1 & 122 & CDK6 & 162 & PTGS1 \\
3 & CA1 & 43 & MMP2 & 83 & PYGL & 123 & CDK2 & 163 & CPA1 \\
4 & CA3 & 44 & PTPN1 & 84 & GSK3B & 124 & ARG1 & 164 & RELA \\
5 & CA6 & 45 & CAPN2 & 85 & SRC & 125 & APP & 165 & TLR9 \\
6 & CA12 & 46 & CAPN1 & 86 & PTK2 & 126 & MCL1 & 166 & PRKCE \\
7 & CA14 & 47 & DAO & 87 & HSD17B2 & 127 & TERT & 167 & F3 \\
8 & CA9 & 48 & ESR2 & 88 & KDR & 128 & TYR & 168 & NOS2 \\
9 & CA5A & 49 & AKR1C1 & 89 & MMP13 & 129 & HSD17B1 & 169 & NGFR \\
10 & CA4 & 50 & AKR1B10 & 90 & MMP3 & 130 & ESRRA & 170 & CCND1 \\
11 & FUT7 & 51 & CHRNA7 & 91 & PLK1 & 131 & PTPRS & 171 & TUBB3 \\
12 & CA13 & 52 & AHR & 92 & CDK1 & 132 & PLG & 172 & ACE \\
13 & CA5B & 53 & RNPEP & 93 & PKN1 & 133 & MPG & 173 & REN \\
14 & TPMT & 54 & IDO1 & 94 & CSNK2A1 & 134 & SLC22A12 & 174 & CTNNB1 \\
15 & AKR1C3 & 55 & MAOB & 95 & MET & 135 & PARP1 & 175 & SLC13A5 \\
16 & TUBB1 & 56 & FOLH1 & 96 & NEK2 & 136 & MMP12 & 176 & ALDH2 \\
17 & POLA1 & 57 & EGFR & 97 & CXCR1 & 137 & CD38 & 177 & PRKCA \\
18 & POLB & 58 & ACHE & 98 & CAMK2B & 138 & TNKS2 & 178 & MMP7
\end{tabular}




\begin{tabular}{|c|c|c|c|c|c|c|c|c|c|}
\hline 19 & SQLE & 59 & NFKB1 & 99 & ALK & 139 & TNKS & 179 & MMP8 \\
\hline 20 & OGA & 60 & MAOA & 100 & AKT1 & 140 & TOP1 & 180 & HSP90AA1 \\
\hline 21 & FYN & 61 & NQO1 & 101 & $\mathrm{ABCB} 1$ & 141 & ESR1 & 181 & SRD5A2 \\
\hline 22 & LCK & 62 & $\mathrm{XDH}$ & 102 & NEK6 & 142 & PTGS2 & 182 & MIF \\
\hline 23 & FBP1 & 63 & CYP1B1 & 103 & PLA2G1B & 143 & CFTR & 183 & SLC6A2 \\
\hline 24 & SERPINE1 & 64 & $\mathrm{ABCC} 1$ & 104 & BACE1 & 144 & AMY1A & 184 & MAPK1 \\
\hline 25 & TTR & 65 & NOX4 & 105 & AXL & 145 & GRK6 & 185 & СТВP2 \\
\hline 26 & KDM4E & 66 & ABCG2 & 106 & NUAK1 & 146 & PFKFB3 & 186 & PIK3CB \\
\hline 27 & KDM4A & 67 & IGF1R & 107 & AKR1C2 & 147 & AR & 187 & CYP2C9 \\
\hline 28 & ERN1 & 68 & ALOX15 & 108 & AKR1C4 & 148 & NMUR2 & 188 & CYP3A4 \\
\hline 29 & $\mathrm{KDM} 2 \mathrm{~A}$ & 69 & ALOX12 & 109 & AKR1A1 & 149 & ADRA2A & 189 & CYP2C19 \\
\hline 30 & KDM4C & 70 & AVPR2 & 110 & GPR35 & 150 & ADRA2C & 190 & PIK3CA \\
\hline 31 & KDM3A & 71 & FLT3 & 111 & MAPT & 151 & RPS6KA3 & 191 & ELANE \\
\hline 32 & KDM6B & 72 & CYP19A1 & 112 & TOP2A & 152 & NQO2 & 192 & COMT \\
\hline 33 & FTO & 73 & $\mathrm{~F} 2$ & 113 & INSR & 153 & PDE5A & 193 & ALDH5A1 \\
\hline 34 & KMO & 74 & PIM1 & 114 & MYLK & 154 & TNF & 194 & ABAT \\
\hline 35 & HCAR2 & 75 & AURKB & 115 & SYK & 155 & IL2 & 195 & CDC25B \\
\hline 36 & AKR1B1 & 76 & DRD4 & 116 & PIK3CG & 156 & SLC29A1 & 196 & NAT1 \\
\hline 37 & SLC16A1 & 77 & ADORA1 & 117 & APEX1 & 157 & NFE2L2 & 197 & ALPG \\
\hline 38 & TLR4 & 78 & GLO1 & 118 & CDK5R1 & 158 & STAT3 & 198 & PLAA \\
\hline 39 & TRPA1 & 79 & MPO & 119 & CDK5 & 159 & HSD11B1 & 199 & PTPRC \\
\hline 40 & ALOX5 & 80 & PIK3R1 & 120 & CCNB1 & 160 & CYP1A1 & 200 & EP300 \\
\hline
\end{tabular}

\subsection{Construction of chemical component-target network diagram}

As shown in Figure 1, the chemical component-action target network diagram involved 214 nodes and 638 edges. The green nodes represent chemical components of RDN and the red nodes represent the targets of the RDN chemical components. Both nodes were connected in a line, representing the direct-action relationship between the two nodes. The top five degree values in the network included isorhamnetin, quercetin, luteolin, ferulic acid, and caffeic acid, suggesting that these components may be the main chemical components of RDN. 


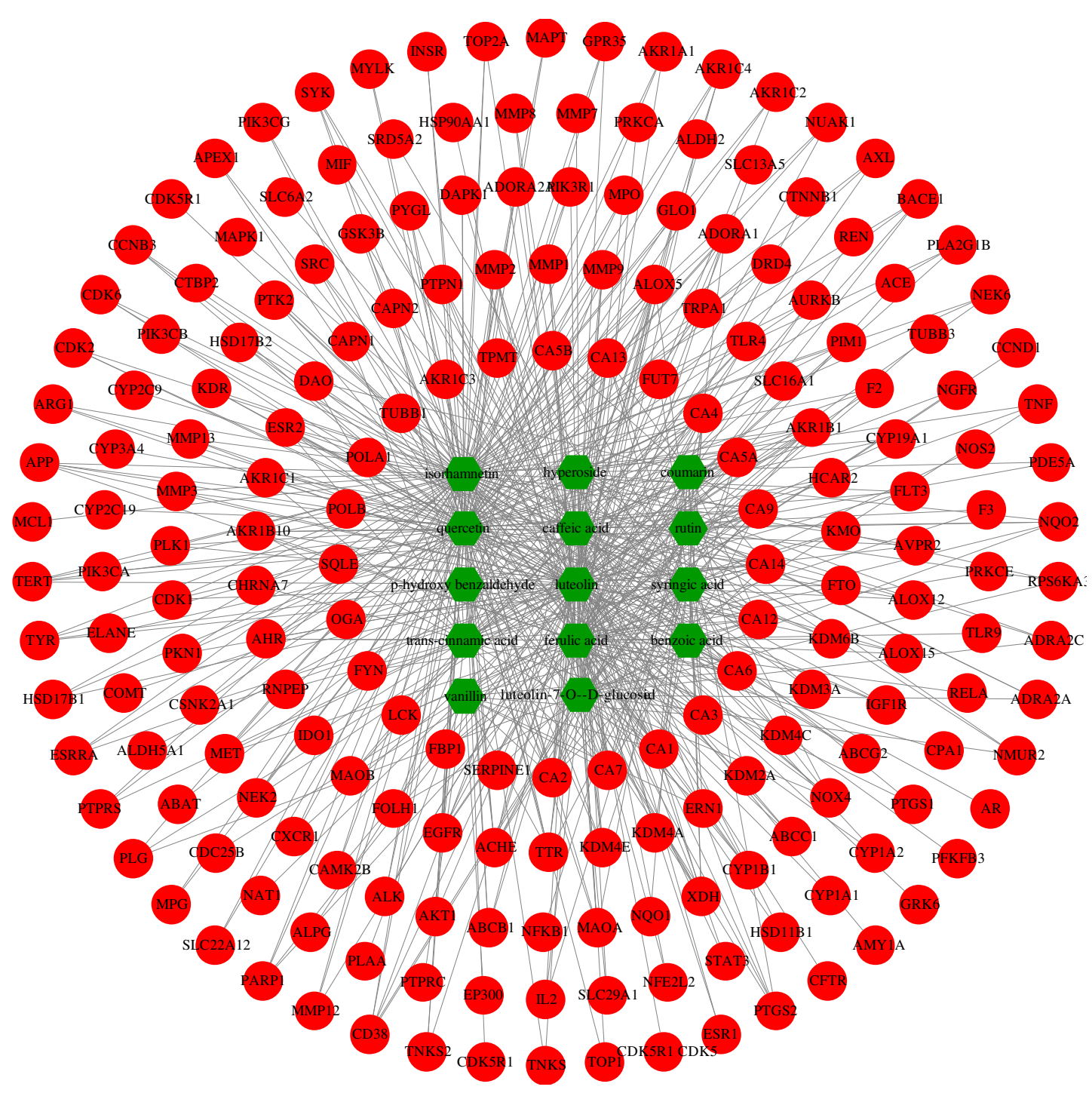

FIGURE 1: Chemical component-target network diagram of RDN.

\subsection{Acquisition of COVID-19 targets and prediction of potential targets for COVID-19 treatment using RDN}

According to the literature, a total of 5,556 genes co-expressed with ACE2 were obtained, and the gene names of the original files were subjected to standardized conversion. A total of 3,558 human-related targets were obtained as potential targets for COVID-19. 54 potential targets of RDN for COVID-19 treatment were obtained by intersecting RDN acting targets with COVID-19 targets (see Figure 2 for details). 


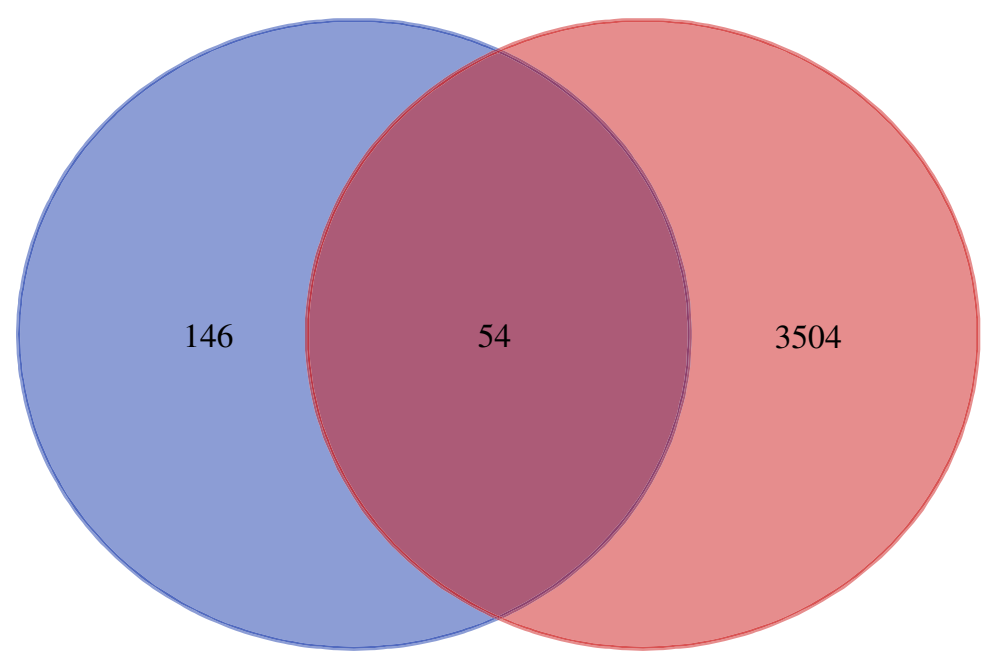

FIGURE 2: Venn diagram of RDN acting targets and COVID-19 targets.

\subsection{Network topology analysis}

After importing the PPI network relationships into the Cytoscape 3.7.0 platform for network topology analysis, 7 targets without network topology analysis attributes were removed, and the final PPI network is shown in Figure 3. The network contains 47 nodes and 142 edges. The nodes in the figure represent the target proteins and the line connecting the two nodes represents the interaction between them. The CytoHubba was used to rank the nodes based on their degree values in the network and to perform visual processing based on the color of the nodes; the brighter the color, the greater the degree value of the node in the network and the more important the node. Among the targets, those with the highest degree values were the epidermal growth factor receptor (EGFR), signal transducer and activator of transcription 3 (STAT3), myeloperoxidase (MPO), estrogen receptor (ESR), ATP-binding cassette superfamily G member 2 (ABCG2), poly [ADP-ribose] polymerase 1 (PARP1), ATP binding cassette subfamily B member 1 (ABCB1), cytochrome P450 family 2 subfamily C member (CYP2C)-19, CYP2C-9, myeloid cell leukemia 1 (MCL1), catenin (cadherin-associated protein), beta 1 (CTNNB1), coagulation factor II, thrombin (F2), and Angiotensin-converting enzyme (ACE), suggesting that these targets may play a key role in RDN treatment of COVID-19. 


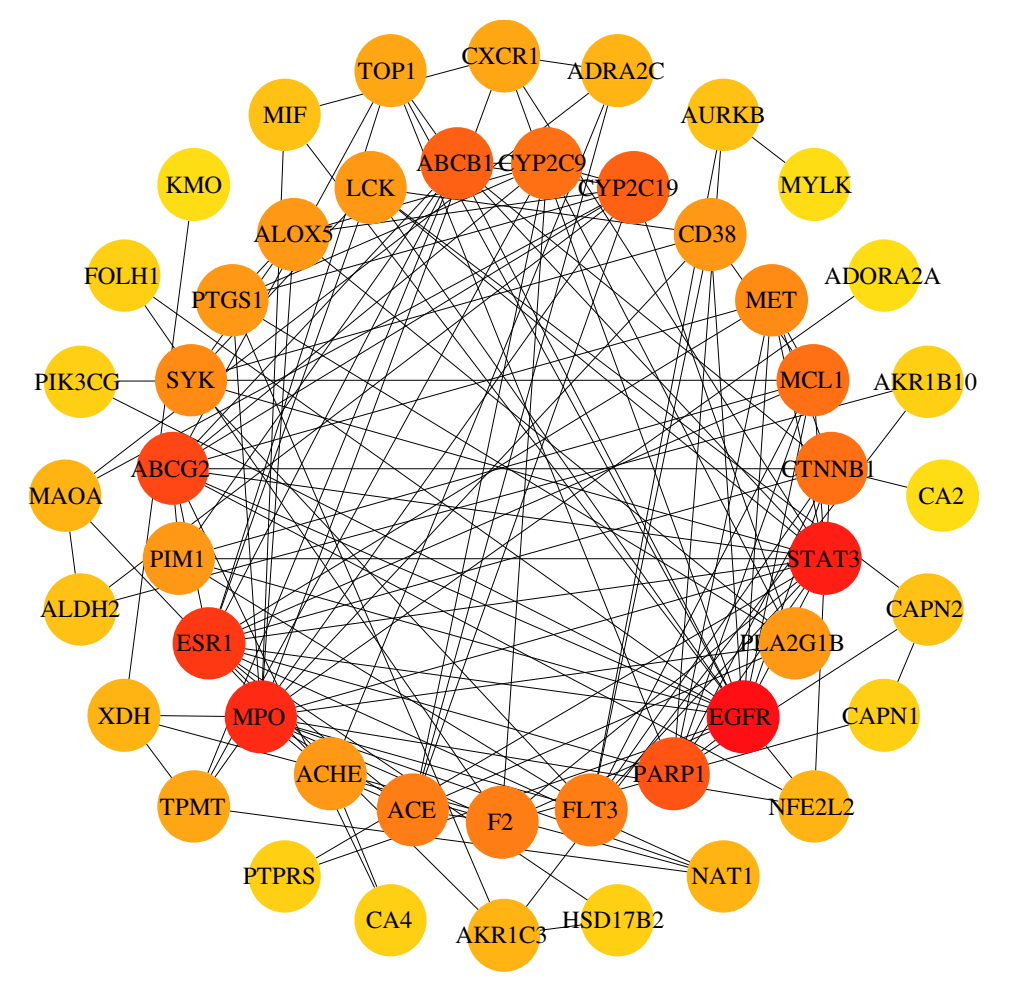

FIGURE 3: PPI networks of potential targets for COVID-19 treatment by RDN.

\subsection{GO functional enrichment analysis}

The biological process (BP) of GO functional enrichment analysis (GO-BP) showed 83 entries, including regulation of vasoconstriction, regulation of cellular response to oxidative stress, arachidonate transport, arachidonic acid secretion, B cell apoptotic process, and regulation of fibroblast proliferation, among others. The molecular function (MF) of GO functional enrichment analysis (GO-MF) showed 17 entries, including oxidoreductase activity, acting on the aldehyde or oxo group of donors, hydro-lyase activity, and positive regulation of phospholipase activity. Relationships between the results of GO-BP and GO-MF and targets were visualized as shown in Figures 4 and 5. The size of the dot reflects $P$ value of the entry; the bigger the size, the smaller the $P$ value and the more important the entry. 


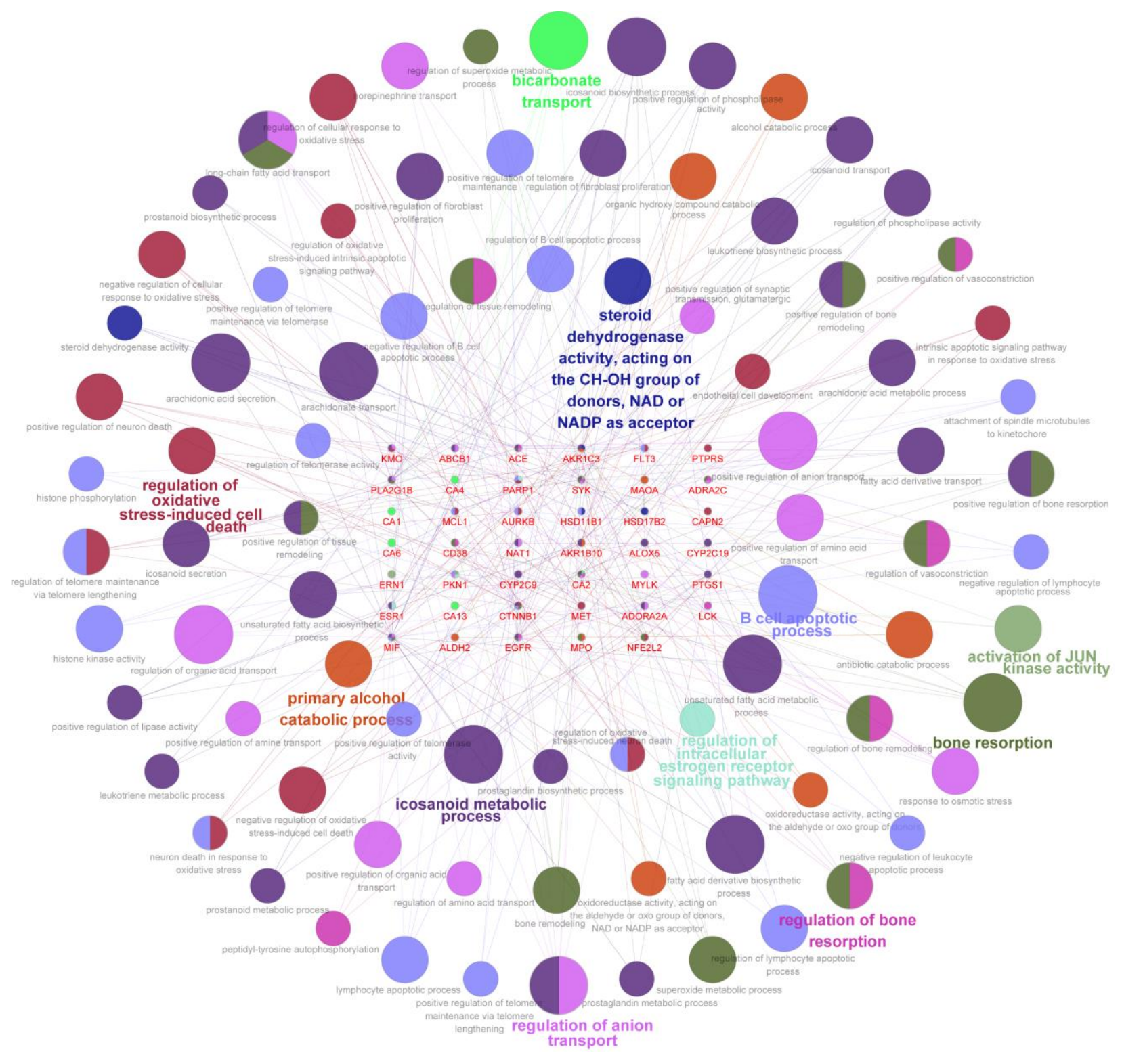

FIGURE 4: GO-BP analysis of potential targets. 


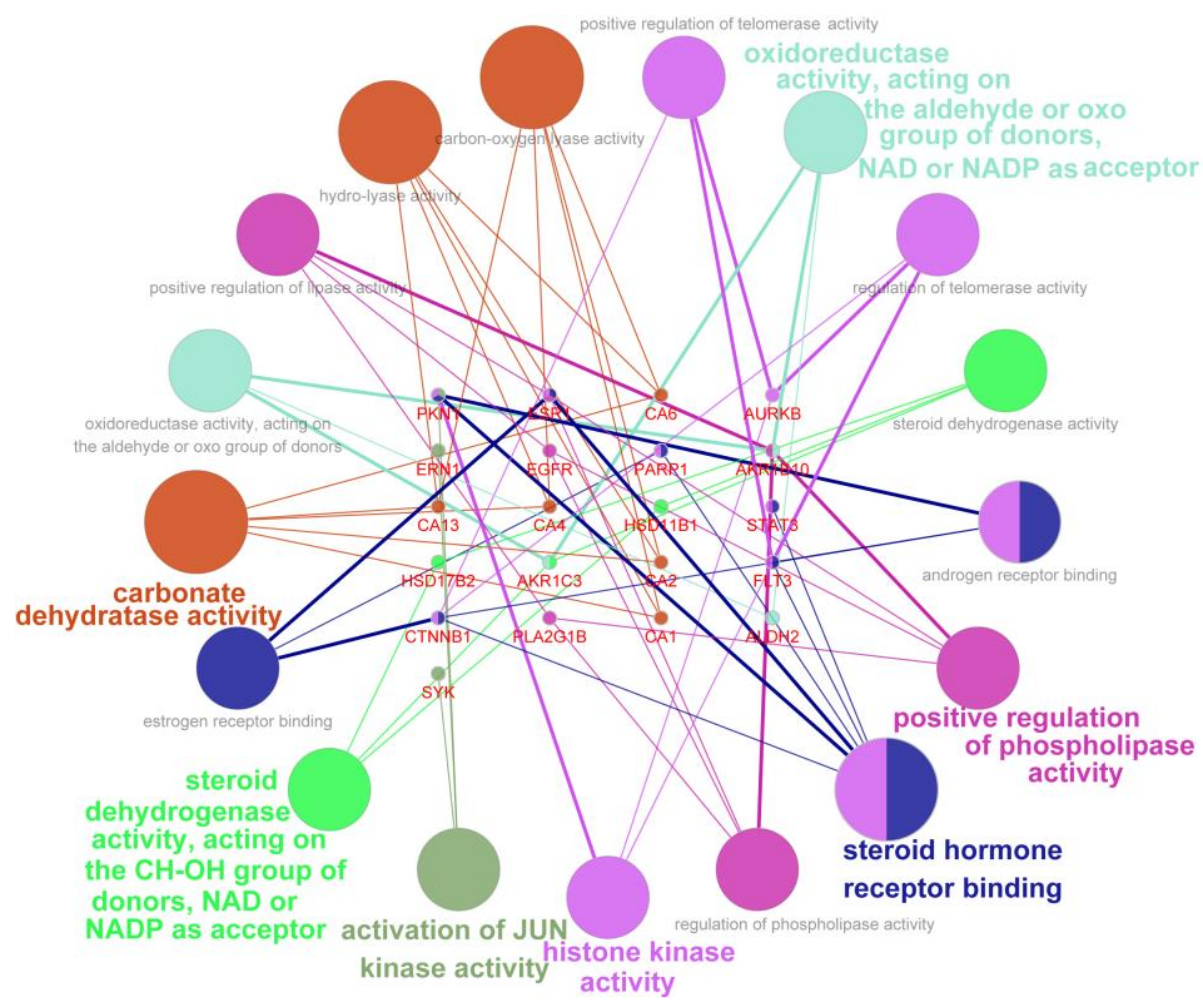

FIGURE 5: GO-MF analysis of potential targets.

\subsection{WikiPathways enrichment analysis}

16 entries, including oxidative stress, interleukin (IL)-5 signaling pathway, and IL-2 signaling pathway were obtained in the WikiPathway enrichment analysis. As shown in Figure 6, the $y$-axis represents the name of the pathway and the $\mathrm{x}$-axis represents the number of genes enriched in the pathway. 


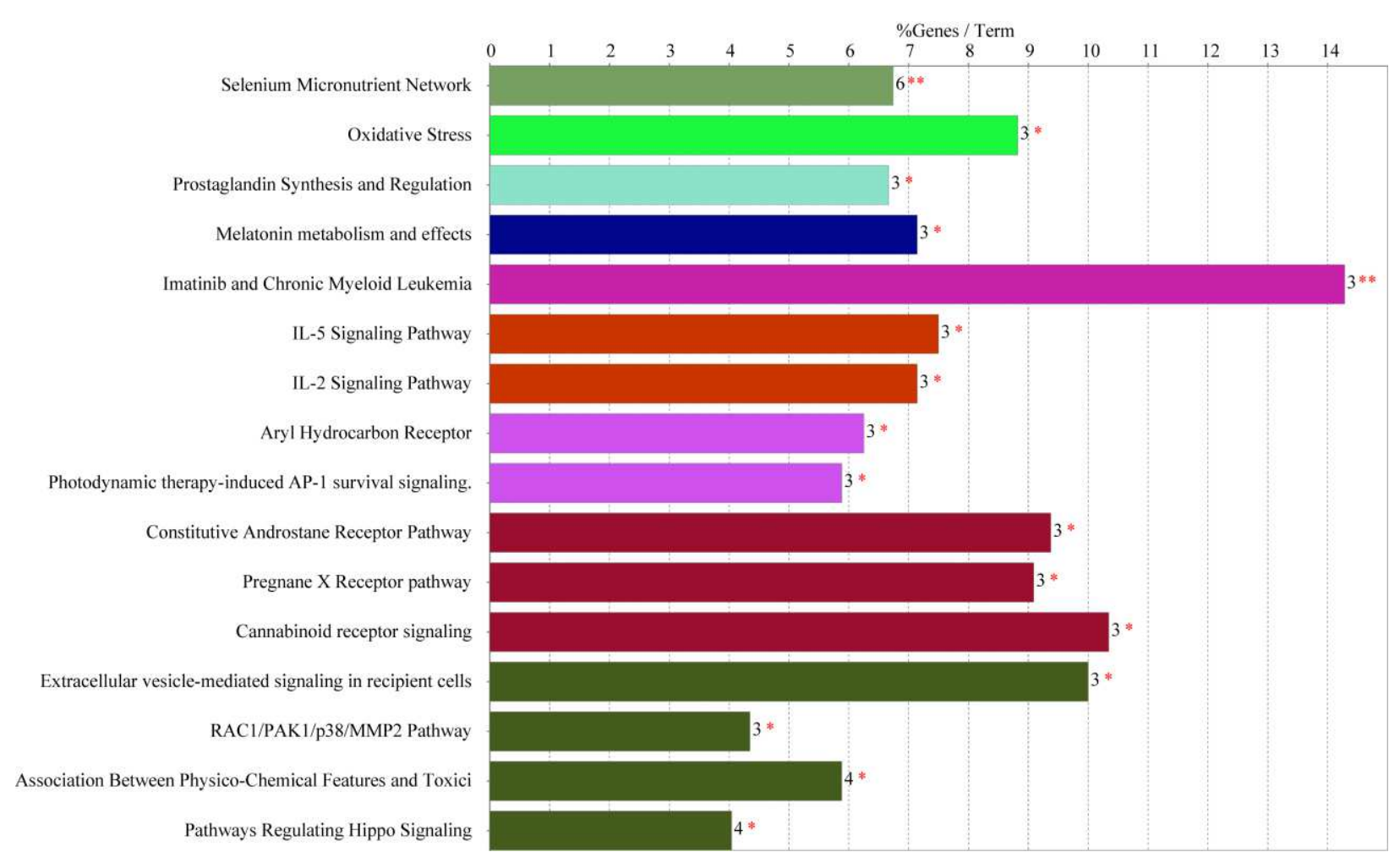

FIGURE 6: WikiPathways enrichment analysis of potential targets.

\section{Discussion}

RDN mainly comprised the flavonoid chemical components of isorhamnetin, quercetin, and luteolin[21]. Isorhamnetin has anti-inflammatory, antiviral, and antioxidant effects, and also affects apoptosis. It significantly lowers the concentrations of IL-6, IL-1 $\beta$, and TNF- $\alpha$ and suppresses the activation of the NF- $\mathrm{kB}$ signaling pathway to exert an anti-inflammatory effect[22]. During inflammation, isorhamnetin also affects apoptosis and autophagy through the P38/peroxisome proliferator-activated receptor-alpha (PPAR- $\alpha$ ) signaling pathway[23]. In addition, isorhamnetin has good efficacy against the influenza virus, and also has antioxidant effects[24, 25].

Quercetin has various effects, including anti-inflammatory, promotion of processes that reduce inflammatory damage and anti-oxidative stress. Quercetin inhibits the expression and secretion of inflammatory apoptosis-inducing factor (AIF) and reduces the inflammatory response of kidney tissues, thus mitigating ischemia-reperfusion injury[26]. It also improves the inflammatory state of the body by reducing the secretion of TNF- $\alpha$ in organs and tissues[27]. Studies have shown that after the activation of $\mathrm{NF}-\kappa \mathrm{B}$ signal transduction pathway, $\mathrm{NF}-\kappa \mathrm{B}$ binds 
to $\mathrm{\kappa B}$ of the inducible nitric oxide synthase (iNOS) gene promoter, leading to the further expression of iNOS and the production of excess nitric oxide (NO), causing a variety of tissue damage and dysfunctions in the body, and quercetin inhibits the activation of the NF- $\kappa \mathrm{B}$ signaling pathway, thereby reducing iNOS expression, avoiding further tissue damage, and improving the pathological state of the disease[28]. Quercetin exerts anti-lipid peroxidation, thereby reducing oxidative stress[29].

Luteolin inhibits angiotensin II-mediated oxidative stress, apoptosis[30], and inflammation, reduces airway mucus secretion, and exhibits antiviral effects. In lipopolysaccharide-induced pneumonia, luteolin reduces apoptosis and inhibits expression of inflammatory cytokines[31]. It also reduces the excessive secretion of mucus in the lung airway epithelium[32]. In addition, luteolin has antiviral properties, which interferes with viral replication in the early stages of influenza A virus infection by inhibiting the expression of its coat protein I complex, thus blocking virus invasion and endocytosis[33]. Luteolin also inhibits the activation of STAT1/3-dependent NF- $\kappa$ B activation, thereby reducing the secretion of the proinflammatory mediator NO, inflammatory cytokines, and the expression of iNOS and cyclooxygenase-2 $(\mathrm{COX}-2)[34]$.

Importantly, the 2003 SARS epidemic was caused by SARS-CoV[35]. The genetic sequence of SARS-CoV-2 was found to be highly homogenous to SARS-CoV[2]. The Coronavirus Study Group once considered this novel coronavirus to be similar to SARS-CoV and named it as SARS-CoV-2[36]. A previous study suggests that 3C-like protease [3CL(pro)] plays a key role in the replication of SARS-CoV and may become a target for SARS-CoV treatment, with quercetin specifically showing an inhibitory effect on 3CL(pro)[37]. Moreover, one study showed that luteolin has anti-SARS-CoV activity that interferes with the process of coronavirus surface antigen binding to receptors and invasion of host cells, playing an antiviral role[38]. These results suggested that quercetin and luteolin may also act on SARS-CoV-2 through the above two mechanisms and play a role in COVID-19 treatment.

The potential targets of RDN mainly included EGFR, STAT3, MPO, and ACE. The EGFR-mediated signaling pathway activates macrophages and cytokine production by activating $\mathrm{NF}-\kappa \mathrm{B}$ and MAPK1/3 signaling pathways, which are considered to be therapeutic targets for the treatment of infectious inflammation[39]. In chronic obstructive pulmonary disease, the signaling pathways involved in EGFR metabolism are activated by overexpression of hypoxia-inducible 
factor 1-alpha (HIF-1 $\alpha$ ), leading to production of a large number of inflammatory cytokines and ultimately, pulmonary inflammation[40]. EGFR is also involved in inflammation and mucus hypersecretion induced by ambient particulate matter[41]. In addition, one study has shown that inhibition of EGFR-related signaling pathways effectively reduces the excessive pulmonary fibrosis response to respiratory viral infections, such as occurs in SARS-CoV infection[42]. Therefore, as a key target of RDN for COVID-19 treatment, EGFR may be associated with improving pulmonary inflammation and reducing excessive airway secretion of mucus and lung tissue fibrosis.

STAT3 has been implicated in a variety of physiological and pathophysiological processes, including inflammatory responses, cell proliferation and differentiation, cell adhesion, tissue regeneration, and angiogenesis[43]. For example, STAT3 affects pro-inflammatory and anti-inflammatory responses under the stimulation of IL-6, IL-10, and other cytokines[44]. In addition, in the acute phase of viral infection, most viruses inhibit STAT3 to reduce the ability of host cells to respond to inflammatory cytokines in the body, while eliminating negative feedback from the antiviral response of the body, suggesting that STAT3 is a potential target for antiviral therapy[43]. It is speculated that RDN may improve the anti-SARS-CoV-2 response of the body by targeting STAT3 and therefore treating COVID-19.

MPO is a heme-containing peroxidase mainly expressed in neutrophils that plays a phagocytic role and promotes both the antibacterial activity of neutrophils and the body's resistance to various pathogens[45]. MPO is also a mediator of the inflammatory response and resulting tissue damage. Increased MPO levels are related to the increase of inflammation and the oxidative stress response[45, 46]. Therefore, RDN may play a role in COVID-19 treatment by affecting MPO's influence on pathogen phagocytosis, inflammation, and oxidative stress.

ACE and its homolog ACE2 are two key enzymes in the renin-angiotensin system (RAS)[47]. ACE helps transform angiotensin (Ang) I into Ang II, while ACE2 reduces Ang II levels[48]. ACE2 has been considered as a receptor for the main host cells of SARS-CoV-2, thus affecting the process of cellular viral infection[49]. SARS-CoV-2 has a higher ACE2 binding capacity than SARS-CoV[50], inferring that SARS-CoV-2 and SARS-CoV are similar and that both can downregulate ACE2 expression after infecting cells[51] to cause an imbalance of ACE2 and ACE in lung tissues, leading to the elevation of Ang II levels. Ang II may cause pulmonary inflammation and lung tissue damage through the angiotensin receptor type 1 (AT1) receptor[52]. 
Therefore, COVID-19 treatment may be closely related to regulating the relationship between ACE2 and ACE imbalance in lung tissue. Combined with the results of this study, we believe these findings show that RDN may regulate the balance of ACE and its homolog ACE2 through ACE targets, thereby reducing pulmonary inflammation and tissue damage.

In the GO functional enrichment analysis, the therapeutic effect of RDN on COVID-19 was mainly related to the regulation of vasoconstriction, regulation of cellular response to oxidative stress, arachidonate transport, arachidonic acid secretion. The WikiPathways enrichment analysis indicated oxidative stress and the IL-5 and IL-2 signaling pathways. After SARS-CoV enters cells, it downregulates the expression of ACE2[51], resulting in increased pulmonary vasoconstriction and permeability, leakage of interstitial fluid, and pulmonary edema[53]. In addition, the formation of oxidative stress and cytokine storms further aggravates lung injury[54], eventually leading to multiple organ failure, including life-threatening respiratory failure. We speculate that SARS-CoV-2 may have a similar mechanism as SARS-CoV. Combined with the results of our GO analyses, we believe that RDN may regulate vasoconstriction and reduce anti-oxidative stress, thereby alleviating the pathological damages in the lung and protecting lung tissues.

Arachidonic acid metabolism is closely related to the production of multiple inflammatory cytokines. Lipoxygenase (LOX) is related to arachidonic acid metabolism and induces the expression of inflammatory cytokines, IL- 6 and TNF- $\alpha$, participates in various inflammatory response processes, and aggravates the local inflammatory response[55]. The literature showed the close relationship between COVID-19 and cytokine storm syndrome[56]. The infiltration of inflammatory cells, alveolar epithelial cell apoptosis, and destruction of the pulmonary capillary barrier structure may lead to pulmonary edema and acute respiratory distress syndrome[57].These findings suggest that RDN may have a potential interventional effect on arachidonic acid metabolism, inhibit the excessive inflammatory response, control cytokine storm syndrome and reduce lung tissue damage and may be significant in restoring the body's normal immune function.

IL-2 is a pleiotropic cytokine produced by antigen activation[58]. IL-2 acts on the IL-2 receptor (IL-2R), thereby forming complex signaling pathways and regulatory networks in the body. After IL-2R is activated, it regulates a variety of physiological activities such as cell proliferation and apoptosis through multiple signaling pathways, including the JAK/STAT, 
PI3K/AKT, and MAPK pathways[59](Figure 7). In addition, IL-2R enhances the immune response of the body and inhibits the activity of autoreactive $\mathrm{T}$ cells, thus maintaining homeostasis[60]. IL-2R is also involved in the differentiation of a variety of helper $\mathrm{T}$ cell subsets. For example, IL-2R signaling enhances the response of T helper type 2 (Th2) cells to IL-4, promotes their differentiation, and enhances host cell defense against the extracellular pathogens[61]. Under the condition of STAT5 phosphorylation, IL-2 induces the proliferation of CD4+ regulatory $\mathrm{T}$ cells[62], suggesting that RDN may participate in the regulation of apoptosis and proliferation, immune response, and other immunological processes through the IL-2 signaling pathway, which may affect the physiological changes seen in COVID-19. 


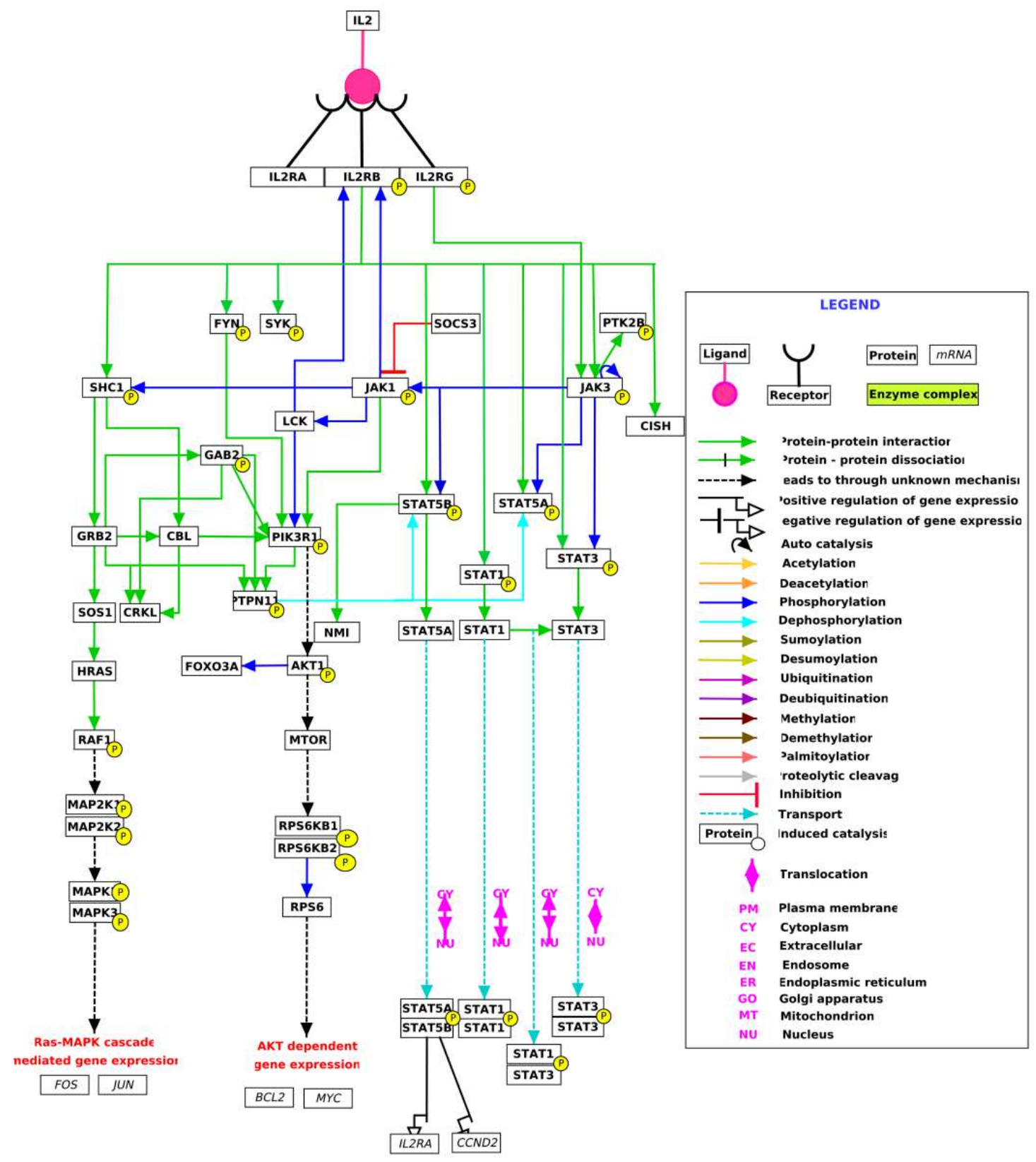

FIGURE 7: Schematic diagram of interleukin (IL)-2 signaling pathway.

\section{Conclusion}

This study used a network pharmacological approach to clarify the specific mechanisms of action of RDN and showed that RDN has multi-component characteristics that could be used to treat COVID-19 in a multi-target, multi-pathway approach. RDN's specific mechanism of action may be centered on its anti-SARS-CoV-2 action, combined with the reduction of inflammatory response, oxidative stress, and lung tissue damage, and the regulation of apoptosis. The 
components, targets, and pathways predicted by this study provide references for the development of clinical treatments and specific, targeted drugs.

However, there are some limitations in the network pharmacology in general. The research model of network pharmacology is based on the known components, targets and pathways. While more and more new components, targets and pathways will be found with the deepening of research. What's more, the results of the study are all predicted by computer. Based on the above limitations, the findings of network pharmacology must be verified by further experimental research.

\section{Data Availability}

The data used during the current study are available from the corresponding author.

\section{Conflicts of Interest}

All the authors declare that they have no conflicts of interest.

\section{Funding}

This project is supported by the National Natural Science Foundation of China (Project No. 81973814), the Guangdong Provincial Traditional Chinese Medicine Inheritance Studio of Professor Xiaohong LIU [Guangdong provincial traditional Chinese medicine office (2018) No. 5], the first class discipline research key project of Guangzhou University of Chinese Medicine (Project No. XK2018014), B3-9 construction project of national clinical medicine research center (respiratory department) (Project No. 2110200309) and the discipline promotion plan of Guangzhou University of Chinese Medicine: the direction of lung disease in internal medicine of traditional Chinese Medicine of Professor Xiaohong LIU (Project No. A3-0402-20-415-008).

\section{Authors' Contributions}

Shaofeng ZHAN proposed the idea and designed the study. Xiaohong LIU revised the protocol. Wenjiang ZHENG and Yong JIANG collected and analyzed the data. Yizi XIE and Xiaohong LIU wrote the paper. All authors contributed to constructive views on the paper. Yizi XIE and Wenjiang ZHENG contributed equally to this work.

\section{References}

1. Zhu N, Zhang D, Wang W et al: A Novel Coronavirus from Patients with Pneumonia in China, 2019. N Engl J Med, 2020; 382(8): 727-33. 
2. Hui DS, E IA, Madani TA et al: The continuing 2019-nCoV epidemic threat of novel coronaviruses to global health - The latest 2019 novel coronavirus outbreak in Wuhan, China. Int J Infect Dis, 2020; 91: 264-66.

3. Wang D, Hu B, Hu C et al: Clinical Characteristics of 138 Hospitalized Patients With 2019 Novel Coronavirus-Infected Pneumonia in Wuhan, China. JAMA, 2020.

4. Guo YR, Cao QD, Hong ZS et al: The origin, transmission and clinical therapies on coronavirus disease 2019 (COVID-19) outbreak - an update on the status. Mil Med Res, 2020; 7(1): 11 .

5. Ren JL, Zhang AH, Wang XJ: Traditional Chinese medicine for COVID-19 treatment. Pharmacol Res, 2020; 155: 104743.

6. Cao ZY, Chang XJ, Zhao ZP et al: Antiviral effects of Reduning injection against Enterovirus 71 and possible mechanisms of action. Chin J Nat Med, 2015; 13(12): 881-8.

7. Jiang $\mathrm{C}$, Zhong $\mathrm{R}$, Zhang $\mathrm{J}$ et al: Reduning injection ameliorates paraquat-induced acute lung injury by regulating AMPK/MAPK/NF-kappaB signaling. J Cell Biochem, 2019; 120(8): 12713-23.

8. Li S, Zhang B: Traditional Chinese medicine network pharmacology: theory, methodology and application. Chin J Nat Med, 2013; 11(2): 110-20.

9. Li H, Yu Y, Wang Z et al: Research on antiviral constituents in Re-Du-Ning Injection ( I ). Chinese Traditional and Herbal Drugs, 2014; 45(12): 1682-88.

10. Daina A, Michielin O, Zoete V: SwissTargetPrediction: updated data and new features for efficient prediction of protein targets of small molecules. Nucleic acids research, 2019; 47(W1): W357-64.

11. Su G, Morris Jh Fau - Demchak B, Demchak B Fau - Bader GD et al: Biological network exploration with Cytoscape 3. Curr Protoc Bioinformatics, 2014; 47: 8-13.

12. Assenov Y, Ramírez F Fau - Schelhorn S-E, Schelhorn Se Fau - Lengauer T et al: Computing topological parameters of biological networks. Bioinformatics, 2008; 24(2): 282-84.

13. Meng Z, Liu X, Wu J et al: Mechanisms of Compound Kushen Injection for the Treatment of Lung Cancer Based on Network Pharmacology. Evid Based Complement Alternat Med, 2019; 2019(Article ID 4637839, 15 pages).

14. Wang J, Zhao S, Liu M et al: ACE2 expression by colonic epithelial cells is associated with 
viral infection, immunity and energy metabolism. medRxiv, 2020: 2020.02.05.20020545.

15. Szklarczyk D, Morris JH, Cook H et al: The STRING database in 2017: quality-controlled protein-protein association networks, made broadly accessible. Nucleic acids research, 2017; 45( D1): D362-68.

16. Chin Ch Fau - Chen S-H, Chen Sh Fau - Wu H-H, Wu Hh Fau - Ho C-W et al: cytoHubba: identifying hub objects and sub-networks from complex interactome. BMC systems biology, 2014; 8 Suppl 4: S11.

17. Bindea G, Mlecnik B Fau - Hackl H, Hackl H Fau - Charoentong P et al: ClueGO: a Cytoscape plug-in to decipher functionally grouped gene ontology and pathway annotation networks. Bioinformatics, 2009; 25(8): 1091-93.

18. Bindea G, Galon J Fau - Mlecnik B, Mlecnik B: CluePedia Cytoscape plugin: pathway insights using integrated experimental and in silico data. Bioinformatics, 2013; 29(5): 661-63.

19. Ashburner M, Ball Ca Fau - Blake JA, Blake Ja Fau - Botstein D et al: Gene ontology: tool for the unification of biology. The Gene Ontology Consortium. Nature genetics, 2000; 25(1): 25-29.

20. Slenter DN, Kutmon M, Hanspers K et al: WikiPathways: a multifaceted pathway database bridging metabolomics to other omics research. Nucleic acids research, 2017; 46(D1): D661-D67.

21. Liu H, Zhang M, Guo Y et al: Solid-phase extraction of flavonoids in honey samples using carbamate-embedded triacontyl-modified silica sorbent. Food Chem, 2016; 204: 56-61.

22. Li Y, Chi G, Shen B et al: Isorhamnetin ameliorates LPS-induced inflammatory response through downregulation of NF-kB signaling. Inflammation, 2016; (1573-2576 (Electronic)).

23. Lu X, Liu T, Chen K et al: Isorhamnetin: A hepatoprotective flavonoid inhibits apoptosis and autophagy via P38/PPAR- $\alpha$ pathway in mice. Biomed Pharmacother, 2018; 103: 800-11.

24. Abdal Dayem A, Choi HY, Kim YB et al: Antiviral effect of methylated flavonol isorhamnetin against influenza. PLoS One, 2015; 10(3): e0121610.

25. Choi YH: The cytoprotective effect of isorhamnetin against oxidative stress is mediated by the upregulation of the Nrf2-dependent $\mathrm{HO}-1$ expression in $\mathrm{C} 2 \mathrm{C} 12$ myoblasts through scavenging reactive oxygen species and ERK inactivation. Gen Physiol Biophys, 2016; 35(2): $145-54$. 
26. Shoskes DA: Effect of bioflavonoids quercetin and curcumin on ischemic renal injury: a new class of renoprotective agents. Transplantation, 1998; 66(2): 147-52.

27. Rivera L, Morón R, Sánchez M et al: Quercetin ameliorates metabolic syndrome and improves the inflammatory status in obese Zucker rats. Obesity (Silver Spring), 2008; 16(9): 2081-7.

28. Dias AS, Porawski M, Alonso M et al: Quercetin decreases oxidative stress, NF-kappaB activation, and iNOS overexpression in liver of streptozotocin-induced diabetic rats. J Nutr, 2005; 135(10): 2299-304.

29. Anjaneyulu M, Chopra K: Quercetin, an anti-oxidant bioflavonoid, attenuates diabetic nephropathy in rats. Clin Exp Pharmacol Physiol, 2004; 31(4): 244-8.

30. Zhang $\mathrm{T}, \mathrm{Wu} \mathrm{W}, \mathrm{Li} \mathrm{D}$ et al: Anti-oxidant and anti-apoptotic effects of luteolin on mice peritoneal macrophages stimulated by angiotensin II. Int Immunopharmacol, 2014; 20(2): 346-51.

31. Liu X, Meng J: Luteolin alleviates LPS-induced bronchopneumonia injury in vitro and in vivo by down-regulating microRNA-132 expression. Biomed Pharmacother, 2018; 106: 1641-49.

32. Shen ML, Wang $\mathrm{CH}$, Lin $\mathrm{CH}$ et al: Luteolin Attenuates Airway Mucus Overproduction via Inhibition of the GABAergic System. Sci Rep, 2016; 6: 32756.

33. Yan H, Ma L, Wang $\mathrm{H}$ et al: Luteolin decreases the yield of influenza A virus in vitro by interfering with the coat protein I complex expression. J Nat Med, 2019; 73(3): 487-96.

34. Liu CW, Lin HW, Yang DJ et al: Luteolin inhibits viral-induced inflammatory response in RAW264.7 cells via suppression of STAT1/3 dependent NF-kappaB and activation of HO-1. Free Radic Biol Med, 2016; 95: 180-9.

35. Hui DSC, Zumla A: Severe Acute Respiratory Syndrome: Historical, Epidemiologic, and Clinical Features. Infect Dis Clin North Am, 2019; 33(4): 869-89.

36. Gorbalenya AE, Baker SC, Baric RS et al: Severe acute respiratory syndrome-related coronavirus: The species and its viruses - a statement of the Coronavirus Study Group. bioRxiv, 2020: 2020.02.07.937862.

37. Nguyen TT, Woo HJ, Kang HK et al: Flavonoid-mediated inhibition of SARS coronavirus 3C-like protease expressed in Pichia pastoris. Biotechnol Lett, 2012; 34(5): 831-8. 
38. Yi L, Li Z, Yuan K et al: Small molecules blocking the entry of severe acute respiratory syndrome coronavirus into host cells. J Virol, 2004; 78(20): 11334-9.

39. Hardbower DM, Singh K, Asim M et al: EGFR regulates macrophage activation and function in bacterial infection. J Clin Invest, 2016; 126(9): 3296-312.

40. Zhang HX, Yang JJ, Zhang SA et al: HIF-1 $\alpha$ promotes inflammatory response of chronic obstructive pulmonary disease by activating EGFR/PI3K/AKT pathway. Eur Rev Med Pharmacol Sci, 2018; 22(18): 6077-84.

41. Wang J, Zhu M, Wang L et al: Amphiregulin potentiates airway inflammation and mucus hypersecretion induced by urban particulate matter via the EGFR-PI3K $\alpha$-AKT/ERK pathway. Cell Signal, 2019; 53: 122-31.

42. Venkataraman T, Frieman MB: The role of epidermal growth factor receptor (EGFR) signaling in SARS coronavirus-induced pulmonary fibrosis. Antiviral Res, 2017; 143: 142-50.

43. Roca Suarez AA, Van Renne N, Baumert TF et al: Viral manipulation of STAT3: Evade, exploit, and injure. PLoS Pathog, 2018; 14(3): e1006839.

44. Gao Y, Zhao H, Wang P et al: The roles of SOCS3 and STAT3 in bacterial infection and inflammatory diseases. Scand J Immunol, 2018; 88(6): e12727.

45. Ndrepepa G: Myeloperoxidase - A bridge linking inflammation and oxidative stress with cardiovascular disease. Clin Chim Acta, 2019; 493: 36-51.

46. Aratani Y: Myeloperoxidase: Its role for host defense, inflammation, and neutrophil function. Arch Biochem Biophys, 2018; 640: 47-52.

47. Gaddam RR, Chambers S, Bhatia M: ACE and ACE2 in inflammation: a tale of two enzymes. Inflamm Allergy Drug Targets, 2014; 13(4): 224-34.

48. Imai Y, Kuba K, Penninger JM: Angiotensin-converting enzyme 2 in acute respiratory distress syndrome. Cell Mol Life Sci, 2007; 64(15): 2006-12.

49. Zhou P, Yang XL, Wang XG et al: A pneumonia outbreak associated with a new coronavirus of probable bat origin. Nature, 2020; 579(7798): 270-73.

50. Wrapp D, Wang N: Cryo-EM structure of the 2019-nCoV spike in the prefusion conformation. Science, 2020; 367(6483): 1260-63.

51. Kuba K, Imai Y, Rao S et al: A crucial role of angiotensin converting enzyme 2 (ACE2) in SARS coronavirus-induced lung injury. Nat Med, 2005; 11(8): 875-9. 
52. Liu L, Qiu HB, Yang Y et al: Losartan, an antagonist of AT1 receptor for angiotensin II, attenuates lipopolysaccharide-induced acute lung injury in rat. Arch Biochem Biophys, 2009; 481(1): 131-6.

53. Imai Y, Kuba K, Rao S et al: Angiotensin-converting enzyme 2 protects from severe acute lung failure. Nature, 2005; 436(7047): 112-6.

54. Channappanavar R, Perlman S: Pathogenic human coronavirus infections: causes and consequences of cytokine storm and immunopathology. Semin Immunopathol, 2017; 39(5): 529-39.

55. Wen Y, Gu J, Chakrabarti SK et al: The role of 12/15-lipoxygenase in the expression of interleukin-6 and tumor necrosis factor-alpha in macrophages. Endocrinology, 2007; 148(3): 1313-22.

56. Huang C, Wang Y, Li X et al: Clinical features of patients infected with 2019 novel coronavirus in Wuhan, China. Lancet, 2020; 395(10223): 497-506.

57. Galani V, Tatsaki E, Bai M et al: The role of apoptosis in the pathophysiology of Acute

Respiratory Distress Syndrome (ARDS): an up-to-date cell-specific review. Pathol Res Pract, 2010; 206(3): 145-50.

58. Liao W, Lin JX, Leonard WJ: Interleukin-2 at the crossroads of effector responses, tolerance, and immunotherapy. Immunity, 2013; 38(1): 13-25.

59. Liao W, Lin JX, Leonard WJ: IL-2 family cytokines: new insights into the complex roles of IL-2 as a broad regulator of $\mathrm{T}$ helper cell differentiation. Curr Opin Immunol, 2011; 23(5): 598-604.

60. Malek TR, Castro I: Interleukin-2 receptor signaling: at the interface between tolerance and immunity. Immunity, 2010; 33(2): 153-65.

61. Zhu J, Yamane H, Paul WE: Differentiation of effector CD4 T cell populations (*). Annu Rev Immunol, 2010; 28: 445-89.

62. Li Y, Liu X, Wang W et al: Low-dose IL-2 expands CD4(+) regulatory T cells with a suppressive function in vitro via the STAT5-dependent pathway in patients with chronic kidney diseases. Ren Fail, 2018; 40(1): 280-88. 


\section{Figures}

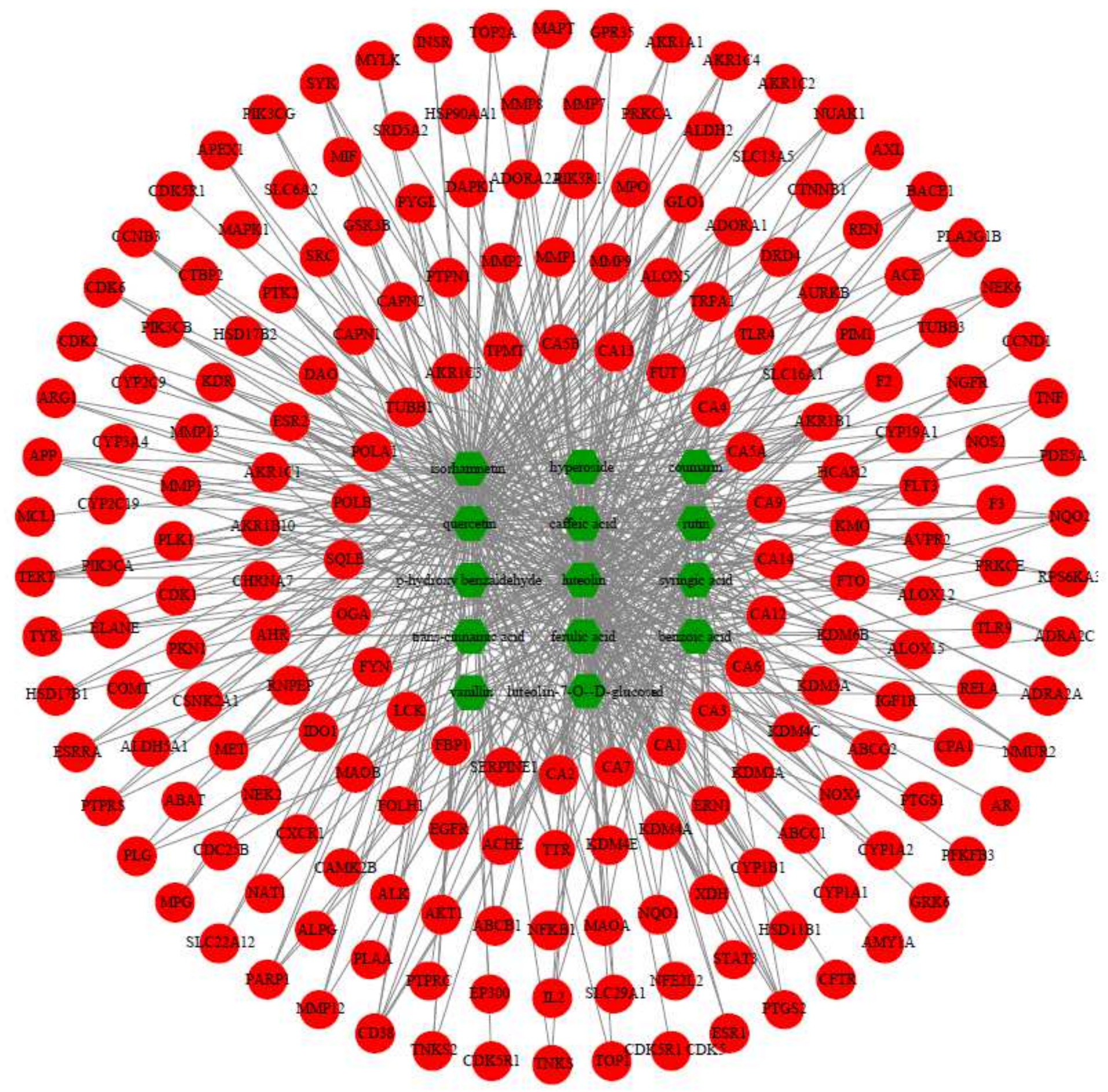

Figure 1

Chemical component-target network diagram of RDN. 


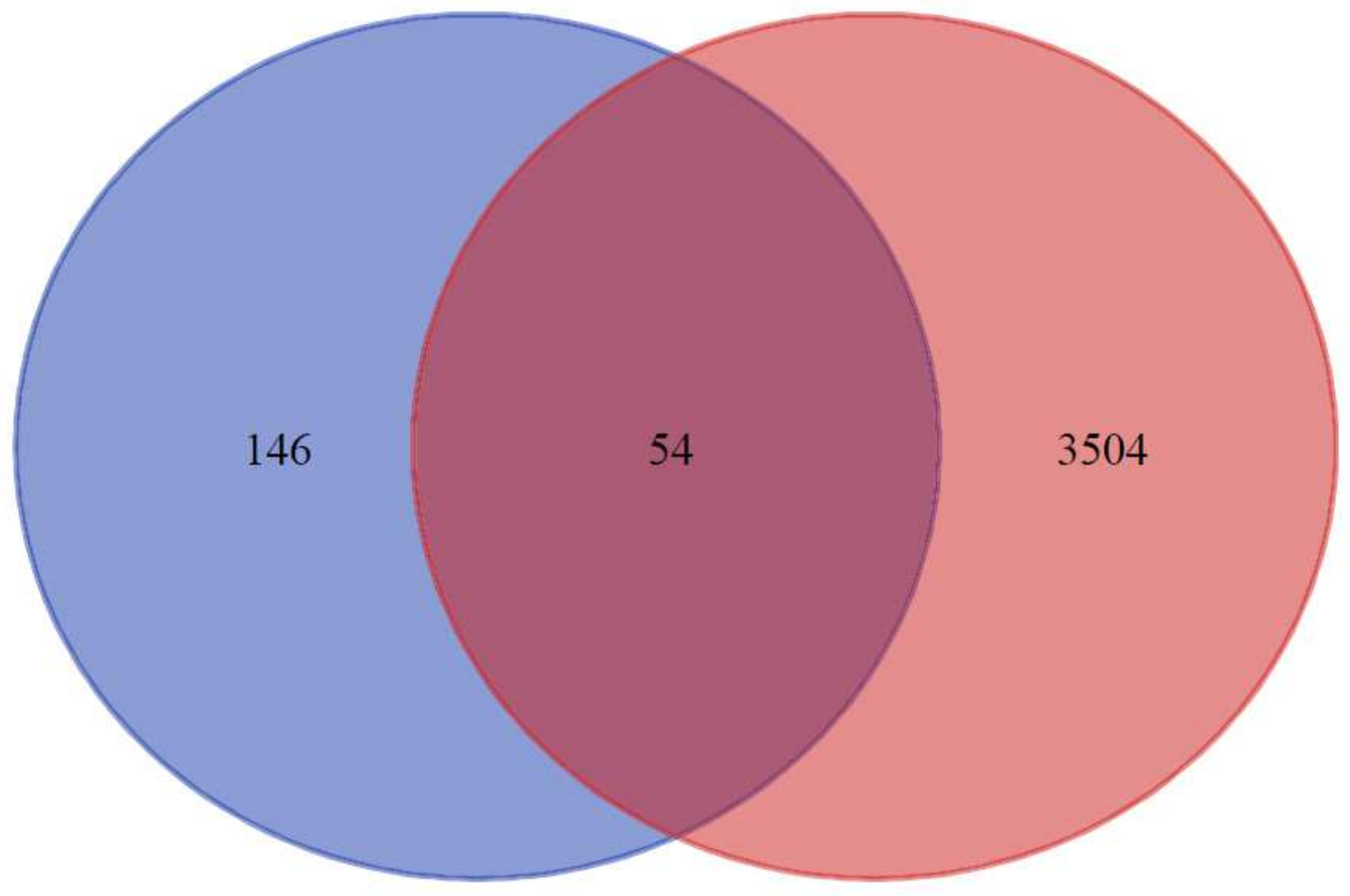

Figure 2

Venn diagram of RDN acting targets and COVID-19 targets. 


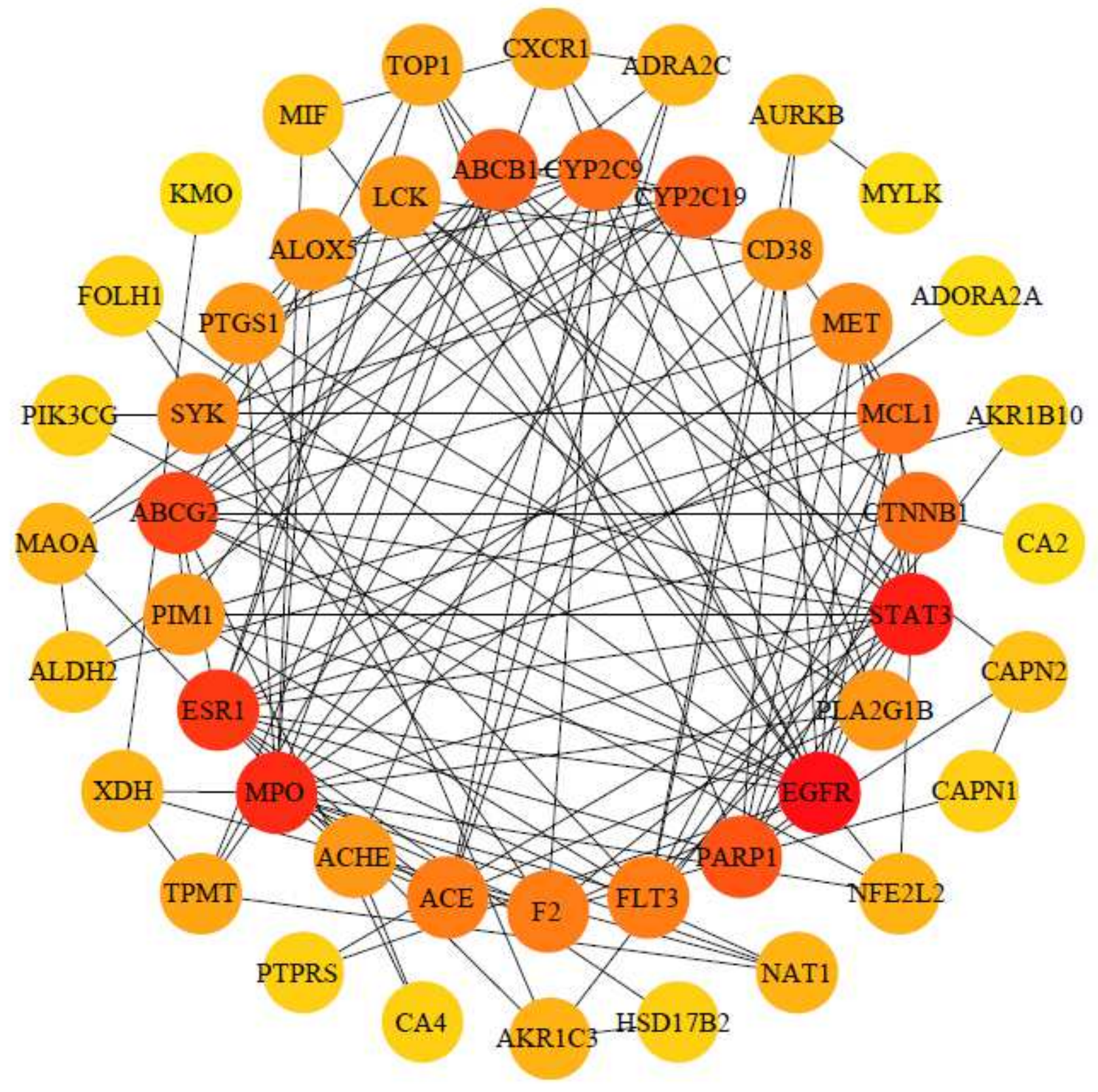

Figure 3

PPI networks of potential targets for COVID-19 treatment by RDN. 


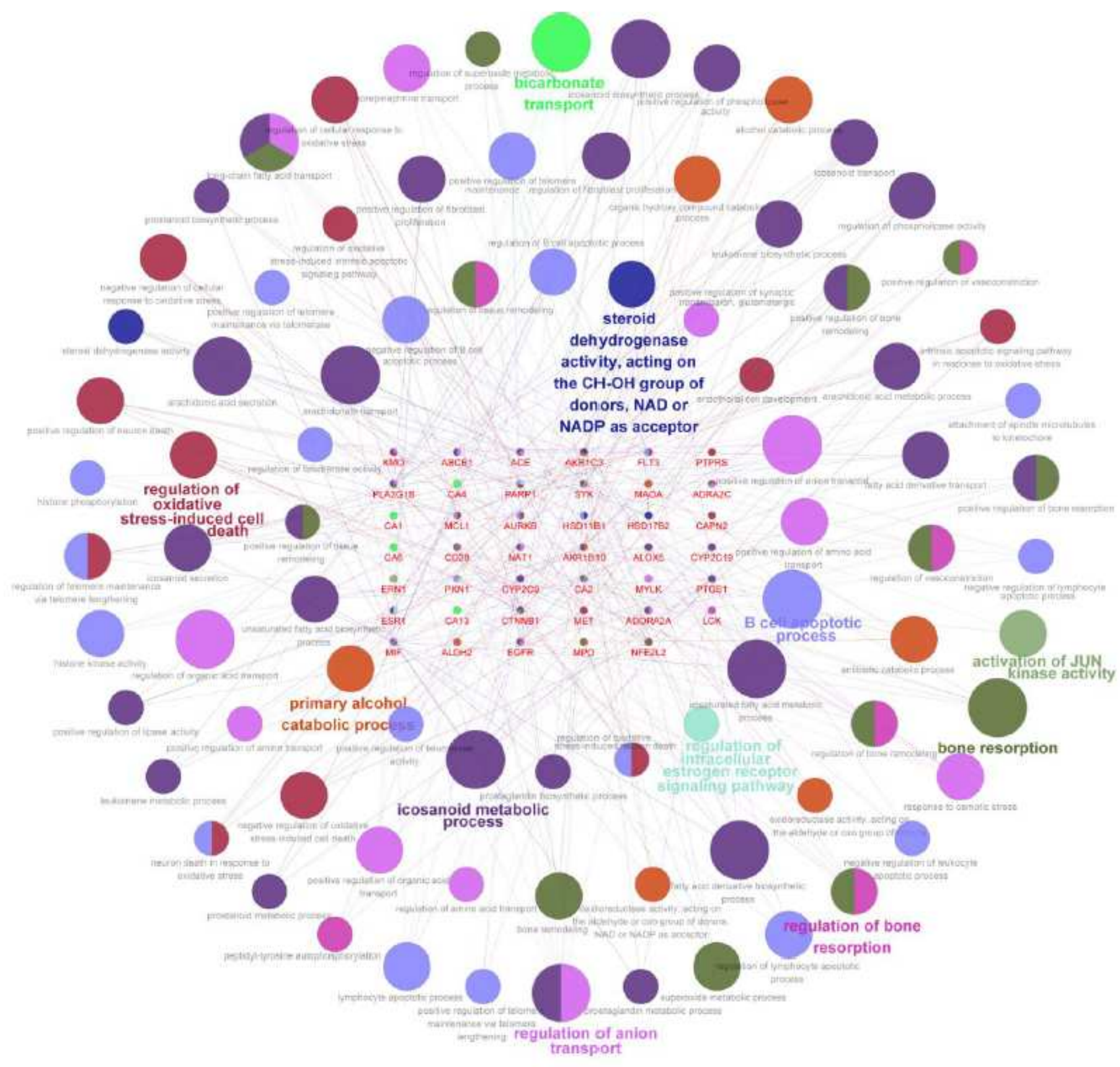

Figure 4

GO-BP analysis of potential targets. 


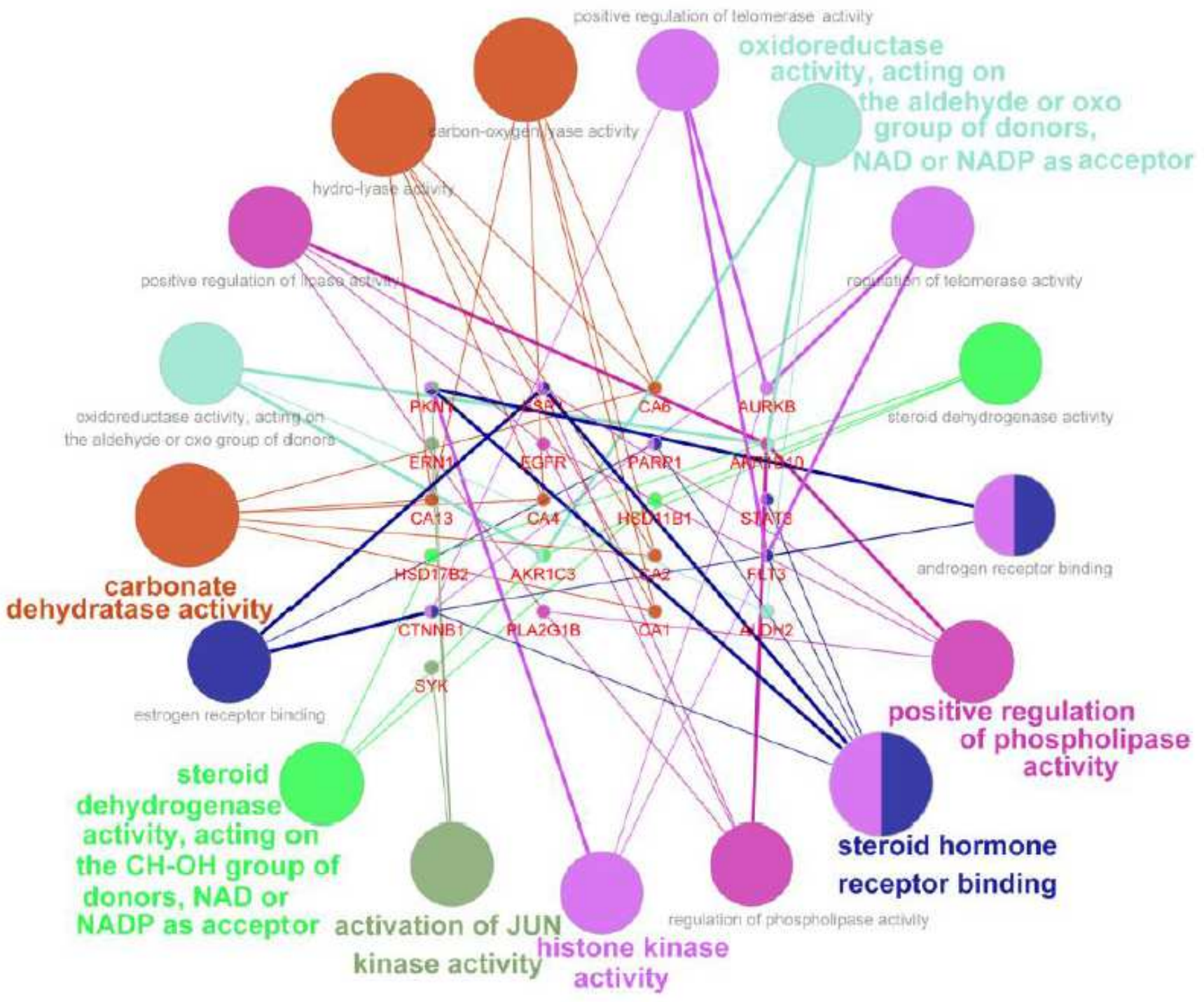

Figure 5

GO-MF analysis of potential targets. 


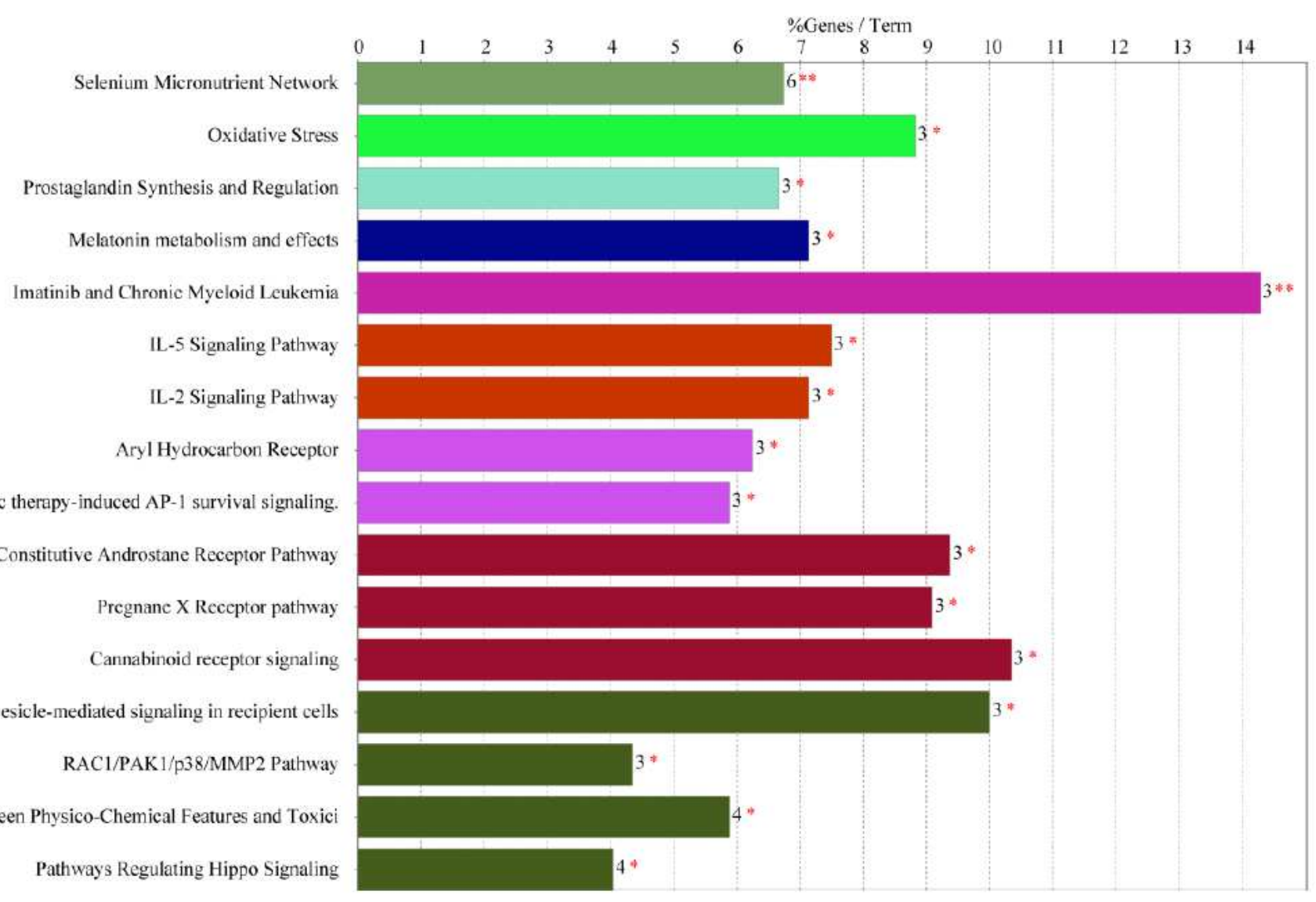

Photodynamic therapy-induced AP-1 survival signaling.

Constitutive Androstane Receptor Pathway

Pregnane X Receptor pathway

Cannabinoid receptor signaling

Extracellular vesicle-mediated signaling in recipient cells

RAC1/PAK1/p38/MMP2 Pathway

Association Between Physico-Chemical Features and Toxici

Pathways Regulating Hippo Signaling

\section{Figure 6}

WikiPathways enrichment analysis of potential targets. 


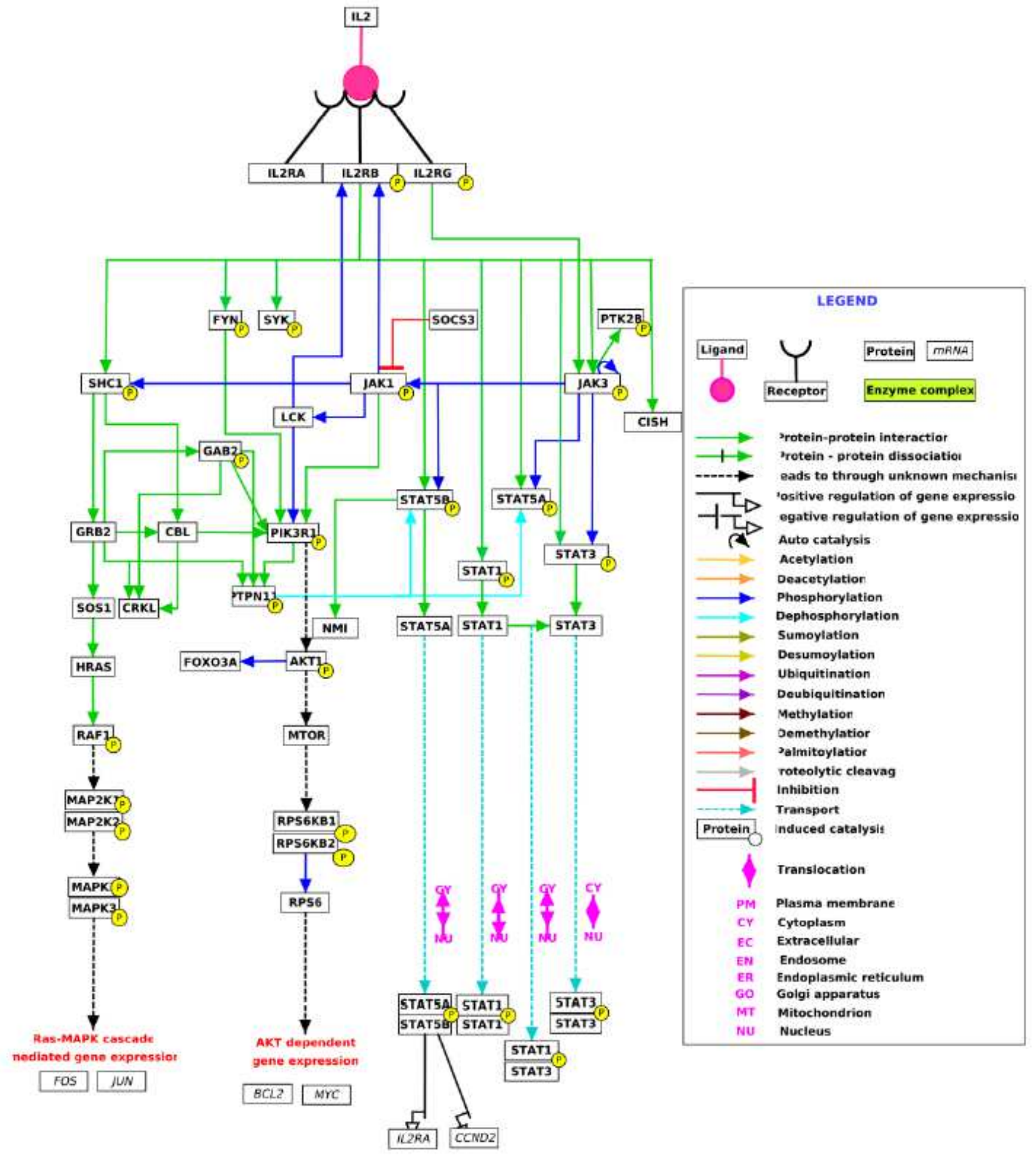

Figure 7

Schematic diagram of interleukin (IL)-2 signaling pathway. 